\title{
Influence of Overburden on Seismic Compression
}

\author{
Yee Eric $^{1, *}$ \\ ${ }^{1}$ KEPCO International Nuclear Graduate School, Department of NPP Engineering, Ulsan, South Korea
}

\begin{abstract}
The state of practice for estimating ground settlements from seismic compression is the simplified procedure. This method was developed in the 1980's and gets its name from the relatively easy method of estimating shear strain demands from potential earthquakes at a site. This procedure is generally split into two parts; a procedure that estimates shear strain demands and a volumetric strain material model. The volumetric strain material model essentially correlates shear strain to resultant volumetric strain. The volumetric strain material model used in the simplified procedure is based on a study from the 1970's, where it was observed that cyclic volumetric strain is independent of vertical stress. Laboratory testing was conducted on a variety of soil materials that are able to experience seismic compression. Reconstituted samples were subjected to cyclic loading in an advanced simple shear testing machine known as the Digitally Controlled Simple Shear device. Factors such as density state and fines content were kept constant across soil categories. Test results show volumetric strain behaviour is dependent on vertical stress and the relationship appears to be power rule based.
\end{abstract}

\section{Introduction}

The initial studies that led to the modern framework on evaluating seismic compression came from researchers Silver and Seed [1]. They conducted strain-controlled simple shear testing with an NGI-type device on dry quartz sand, Crystal Silica No. 20 to be exact. The researchers observed that volumetric strain was controlled by relative density, cyclic shear strain amplitude, and the number of uniform shear strain cycles. The researchers also found that volumetric strain was not significantly influenced by confining stress as shown in Figure 1 .

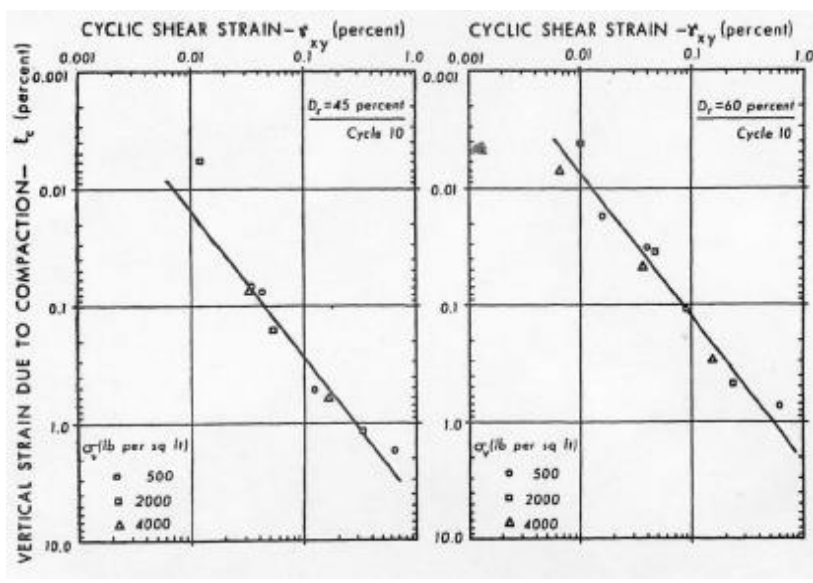

Fig. 1. Effect of surcharge on vertical strain [1].

Shortly afterwards, another researcher confirmed these findings with Ottawa sand and also showed that volumetric strain was independent of loading frequency and saturation level [2]. The work also demonstrated a lack of volume change at cyclic shear strains of less than $0.01 \%$. This limiting value would later be defined as the volumetric threshold shear strain with a recommended range of $0.01-0.03 \%$ for sands $[3,4]$.

Seed and Silver later utilized the results from Silver and Seed and proposed a procedure for estimating the settlement of dry sands during earthquakes that required a site response analysis [5]. The researchers conducted shake table tests on sandy layers of different relative densities, with some of the results shown in Figure 2, to validate their procedure. The figure shows the effect of surcharge loading in those tests, as the specimen with surcharge settled much less than the specimen without. Nonetheless, confining pressure was not recommended as a controlling parameter in the analysis procedure.

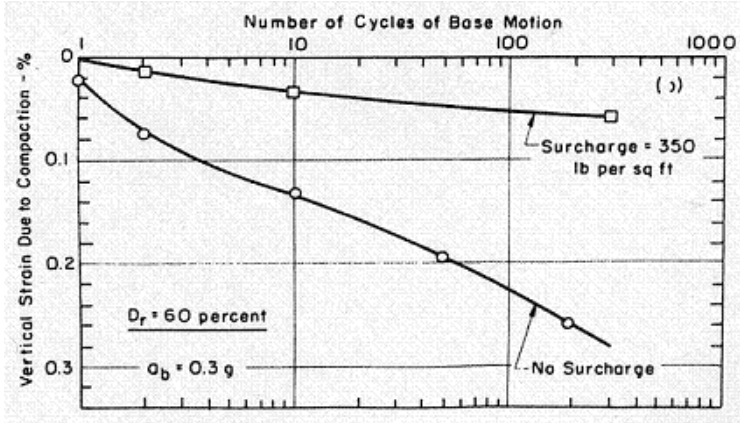

Fig. 2. Effect of surcharge on vertical strain [5].

Subsequently, the Seed and Silver procedure would be modified to include multidirectional shaking effects $[5,6]$. These effects of multidirectional shaking were observed

\footnotetext{
* Corresponding author: eric.yee@kings.ac.kr
} 
from shake table testing of Monterey No. 0 sand. They found the total settlement from cyclic loading was approximately equal to the sum of the settlements caused by the components acting separately. As an example, if a ground motion was decomposed into north-south and east-west components, the resultant settlement from both of those motions acting simultaneously would be approximately equal to the resultant settlement due to shaking from the north-south ground motion and the resultant settlement due to shaking from the east-west ground motion.

Using the laboratory test data on dry clean sand by Silver and Seed [1], Tokimatsu and Seed proposed a procedure for estimating settlements from seismic compression [1,7]. This procedure linked standard penetration test blow count $\left(N_{1}\right)_{60}$, shear strain for a magnitude 7.5 earthquake and volumetric strain. Adjustments are made for earthquakes of differing magnitude through a number of equivalent cycles [8]. They also recommended doubling the estimated settlements to account for multidirectional shaking effects [6,7]. These relationships are part of a volumetric strain material model, VSMM, which correlates shear strain as input and volumetric strain as output. This procedure would later be known as the simplified procedure because of its relatively simple approach to estimating shear strain demands without the direct use of ground response analyses. Although the simplified procedure takes into account total stress as overburden on the demand side, that is, in the calculation of equivalent shear strain, it does not appear to consider overburden on the capacity side, that is, the VSMM used in the simplified procedure has no direct input for overburden. This paper will present the inclusion of overburden as a VSMM component in seismic compression studies.

\section{Methodology}

To study the effects vertical stress may have on seismic compression behaviour, cyclic simple shear testing was conducted on reconstituted sandy soil specimens from bulk samples. The cyclic simple shear device used was the digitally controlled simple shear device at the University of California, Los Angeles that was specifically designed to measure volume change due to shear straining [9].

As an example, bulk soil samples for testing were taken from the Newhall site located in the Greater Los Angeles area. This soil will be called Newhall\#2 and has a natural fines content, $F C=14$, which is the percentage weight of fines in the soil matrix. The fines have a liquid limit of 26 and a plasticity index of 7 . Reconstituted specimens were made by mixing a preset percentage of fines with the base sand for $F C=0,10$, and 50 . However, density state was an issue since relative density, $D_{\mathrm{R}}$, is typically used for clean sands and relative compaction, $R C$, is practically used for all other mixtures. A testing program was conducted to evaluate the relationship between $D_{\mathrm{R}}$ and $R C$ for Newhall\#2 and is shown in Figure 3.

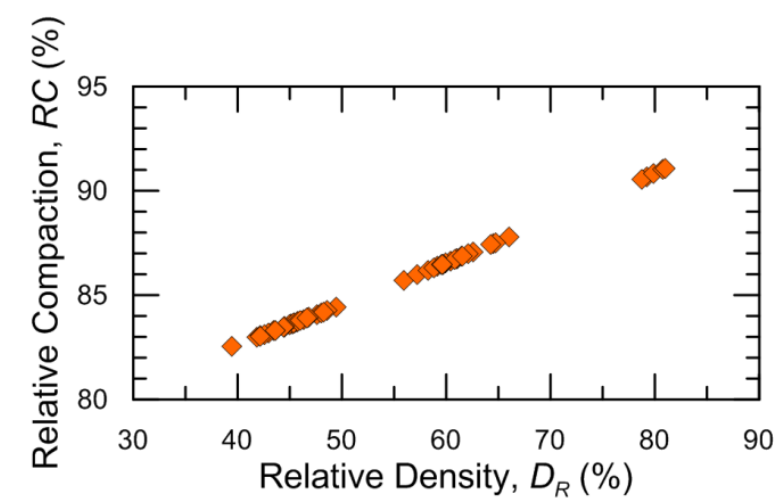

Fig. 3. Correlation between relative density and relative compaction for Newhall\#2.

Based on these test results, the following relationship was developed:

$$
R C=74+0.20 D_{\mathrm{R}}
$$

These specimens were confined laterally by a wirereinforced membrane with a diameter of $102 \mathrm{~mm}$ and were prepared to a variety of density states following standardized protocols [10]. The height of each specimen was varied to reach target density state after being consolidated under vertical stresses of 50,100,200, or $400 \mathrm{kPa}$. Afterwards, specimens were sheared at constant displacement amplitudes resulting in shear strains, $\gamma_{c}=$ $0.01-1 \%$ and at a loading frequency of $1 \mathrm{~Hz}$. To ensure data fidelity, only one test was conducted for each specimen.

Figure 4 summarizes the results from simple shear testing, relating vertical strain after 15 cycles, $\left(\varepsilon_{\mathrm{v}}\right)_{\mathrm{N}=15}$, with constant shear strain amplitude, $\gamma_{c}$. Power law fits to the data were correlated with [10]:

$$
\begin{gathered}
\left(\varepsilon_{\mathrm{v}}\right)_{\mathrm{N}=15}=a\left(\gamma_{\mathrm{c}}-\gamma_{\mathrm{tv}}\right)^{b} \\
C_{\mathrm{N}}=\left(\varepsilon_{\mathrm{v}}\right)_{\mathrm{N}} /\left(\varepsilon_{\mathrm{v}}\right)_{\mathrm{N}=15}=1+R \ln (N / 15)
\end{gathered}
$$

where $a, b$, and $R$ are regression coefficients $\gamma_{\mathrm{tv}}$ is the volumetric threshold shear strain estimated from test data using semi-computational procedures [11], and $N$ is the number of equivalent loading cycles. The parameterization in Eq. (2) however, does have simple explanations. Parameter $b$ could be treated as the slope of the power law fit through the test data while parameter $a$ can be treated as the vertical strain at approximately $1 \%$ cyclic shear strain. An example of how what these parameters mean is also shown in Figure 4. The figure also indicates the regressed parameters $a$ and $b$ for two different relative densities, which are consistent with previous observations that an increase in relative density with lead to a decrease in volumetric strain. After an examination of the data, it was observed that parameter $b$ averaged to about 1.2 and changes to this parameter value did not have a significant impact on the regression [10]. Therefore, parameter $b$ will be treated as 1.2 for the analyses from hereafter. 


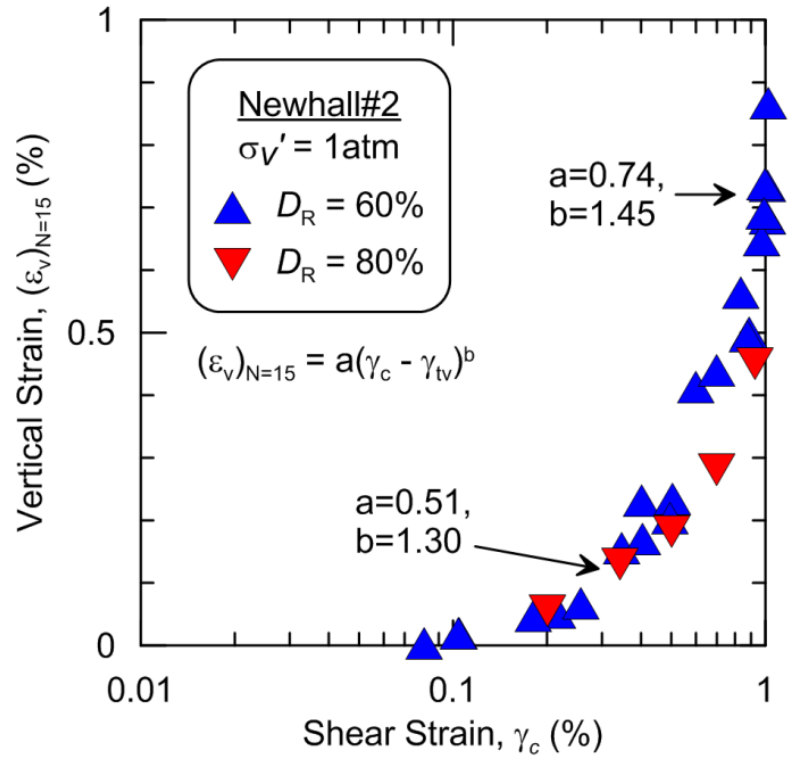

Fig. 4. Example simple shear test data after 15 cycles of constant amplitude shearing.

The parameterization in Eq. (3) defines the cycle to cycle variability in seismic compression behaviour and is normalized to 15 cycles in this representation with parameter $\mathrm{R}$ being the slope of the data in log cycle space. Figure 5 shows an example of how this parameterization manifests itself in the cyclic simple shear data set. The figure shows much of the cycle to cycle variability occurs in the first 3 to 4 cycles and then appears to stabilize to a constant slope.

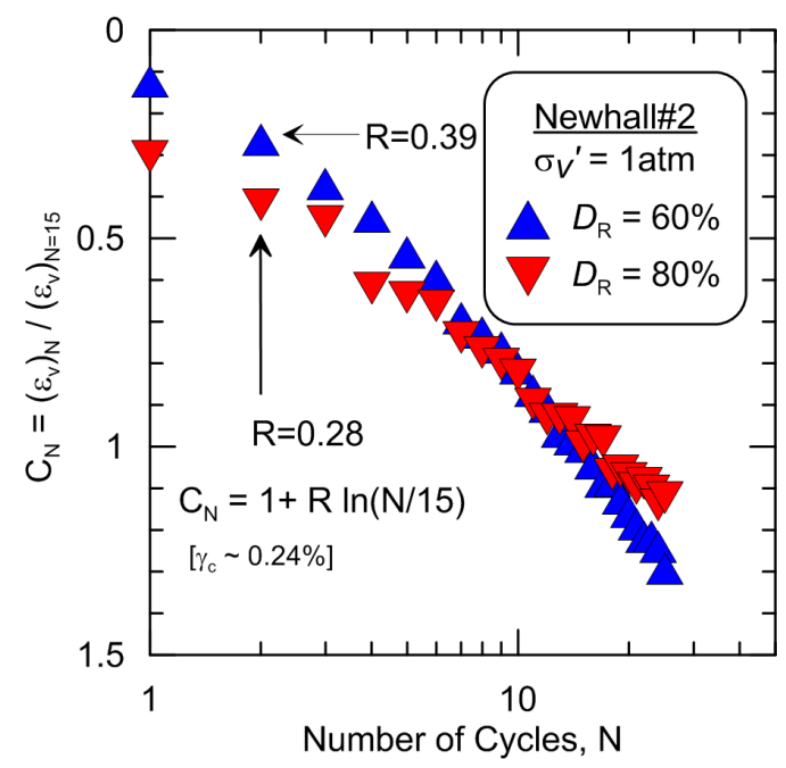

Fig. 5. Example simple shear test data showing cycle to cycle variability.

\section{Overburden}

To investigate the effects of overburden pressure, additional parameterization was needed. Following research in the areas of liquefaction, another parameter was utilized. Intercept parameters within the same density state were normalized to $K_{\sigma, \varepsilon}=(\mathrm{a})_{\sigma} /(\mathrm{a})_{\sigma=1 \mathrm{~atm}}$, where $(\mathrm{a})_{\sigma}$ $=1 \mathrm{~atm}=$ parameter $a$ under $1 \mathrm{~atm}$ of vertical stress, approximately $100 \mathrm{kPa}$, and $(\mathrm{a})_{\sigma}=$ parameter $a$ under another vertical stress. Figure 6 shows $K_{\sigma, \varepsilon}$ versus normalized vertical stress, $\sigma_{\mathrm{v}} / \mathrm{P}_{\mathrm{a}}$, where $\mathrm{P}_{\mathrm{a}}=$ atmospheric pressure. Figure 6 shows the results of this parameterization for varying $F C$ and $R C$.

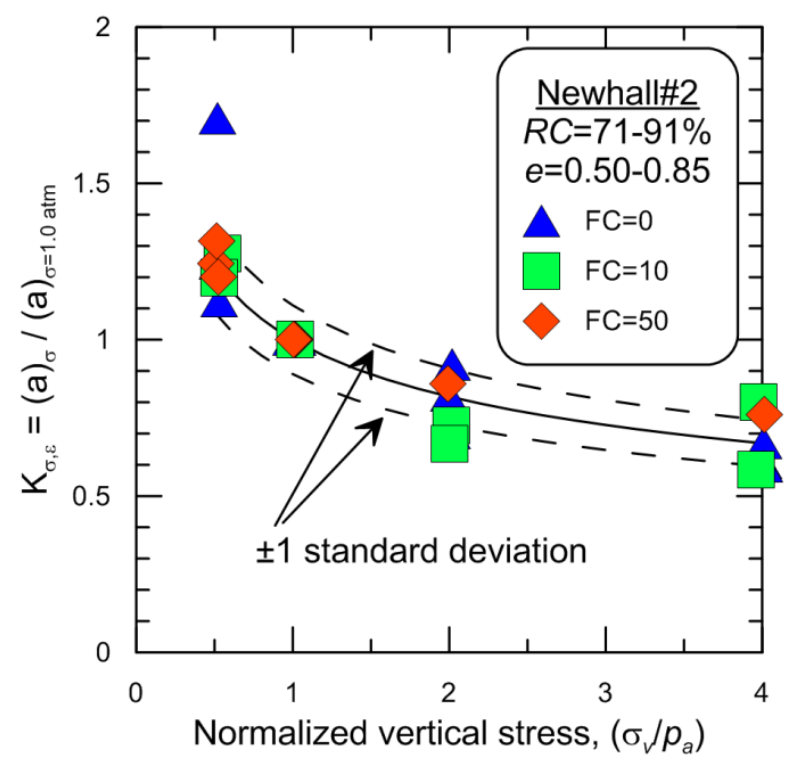

Fig. 6. Example simple shear test data after 15 cycles of constant amplitude shearing.

Figure 6 shows a functional dependence on vertical stress. This observation is different from previous research where investigators reportedly found no dependence on overburden pressure in clean sands [1,2]. Figure 6 also shows the overburden correction factor and the uncertainty expressed in standard deviation. This relationship is shown as:

$$
K_{\sigma, \varepsilon}=(\mathrm{a})_{\sigma} /(\mathrm{a})_{\sigma=1 \mathrm{~atm}}=\left(\sigma_{\mathrm{v}} / \mathrm{P}_{\mathrm{a}},\right)^{-0.29}
$$

These findings can be interpreted through classical particle interaction concepts. By increasing the vertical stress on the soil sample, internal normal stresses between particles are also increased and as a result, an increase in interparticle contact areas. Due to increased interparticle contact areas, a larger fraction of the induced shear strains is used towards reducing the interparticle contact areas until plastic deformation can occur. Since vertical strains from seismic compression are a result of plastic deformations, the increase in vertical stress should decrease vertical strain under a given level of cyclic shear deformation. Moreover, Figure 6 shows no significant dependence on $F C$ or density.

Another component of the VSMM is the relationship defining cycle to cycle variability. A plot of normalized vertical stress against parameter $R$, the parameter that characterizes the cycle to cycle vertical strain relative to the vertical strain after 15 cycles, is shown in Figure 7. The figure does not appear to show any significant dependence on vertical stress. 


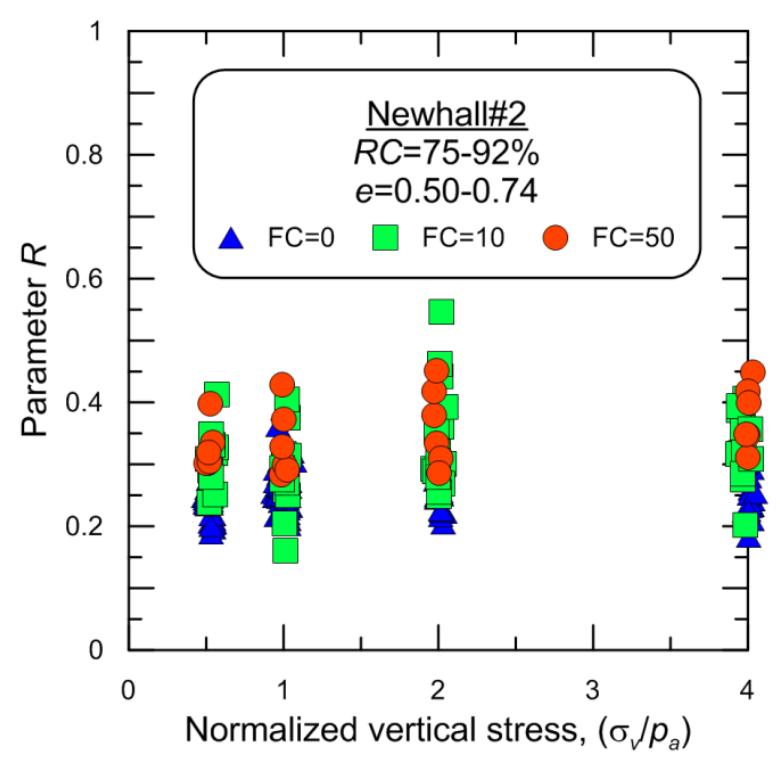

Fig. 7. Example simple shear test data after 15 cycles of constant amplitude shearing.

However, when the data is plotted in a format that compares parameter $R$ with the cyclic shear strain, there appears to be a slight relationship as shown in Figure 8. The data suggest, that for the tested levels of overburden, parameter $\mathrm{R}$ starts at about 0.40 near the threshold shear strain and converges to approximately 0.29 quite quickly as cyclic shear strain increases.

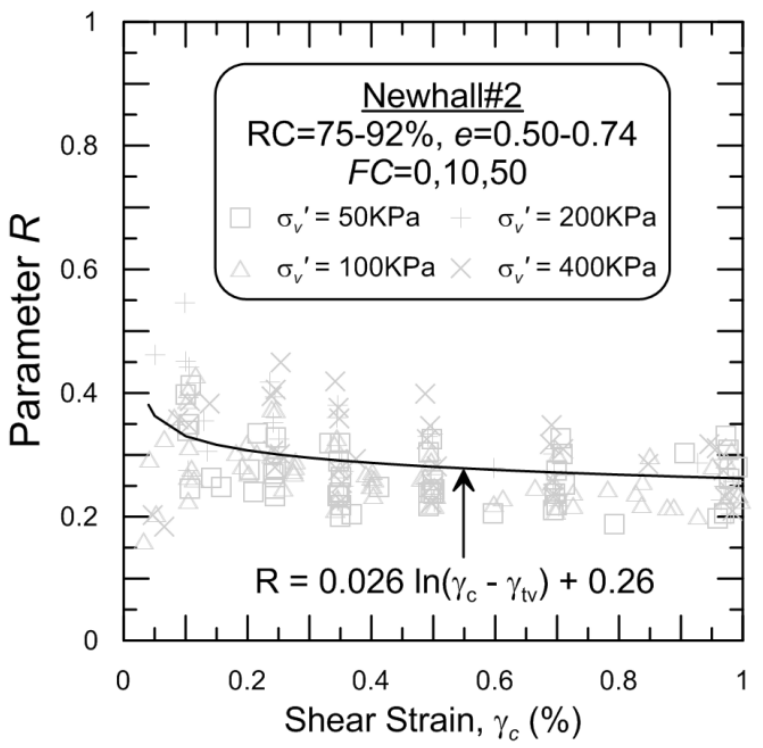

Fig. 8. Example simple shear test data after 15 cycles of constant amplitude shearing.

Figure 8 also shows this strain dependent correction factor and the uncertainty expressed in standard deviation. This relationship is shown as:

$$
R=0.026 \ln \left(\gamma_{\mathrm{c}}-\gamma_{\mathrm{tv}}\right)+0.26
$$

\section{Conclusions}

Previous work in the 1970's that investigated seismic compression behaviour reported that there was no significant dependence on overburden. An experimental program utilizing a special cyclic simple shear machine to measure vertical strains on a soil material, Newhall\#2, while being shook. Density state was addressed by correlating relative density and relative compaction to facilitate a proper comparison. Results showed an inverse power law correlating vertical stress to an intermediate factor relating the vertical strain at a vertical stress of 100 $\mathrm{kPa}$ with other levels of vertical stress. Additionally, the effect of overburden on cycle to cycle variability was also analyzed. There did not appear to be a significant relationship between vertical stress and cycle to cycle behaviour, but the process showed a dependence of cycle to cycle behaviour to cyclic shear strain demand. These observations were explained through the use of modulus and interparticle contacts, where an increase in overburden and confining stress would increase interparticle contact areas, making straining more difficult, and thus a higher modulus.

This work was supported by the 2019 Research Funds of the KEPCO International Nuclear Graduate School (KINGS), Republic of Korea.

\section{References}

1. M.L. Silver, and H.B. Seed, Journal of Soil Mechanics and Foundations Division, 97(9), 11711182, (1971)

2. T.L. Youd, Journal of Soil Mechanics and Foundations Division, 98(7), 709-725, (1972)

3. M. Vucetic, Journal of Geotechnical Engineering, 120(12), 2208-2228, (1994)

4. C-C. Hsu, and M. Vucetic, Journal of Geotechnical and Geoenvironmental Engineering, 130(1) 58-70, (2004)

5. H.B. Seed, and M.L. Silver, Journal of Soil Mechanics and Foundations Division, 98(4), 381397, (1972)

6. R. Pyke, H.B. Seed, and C.K. Chan, Journal of Geotechnical Engineering, 101(4), 379-398, (1975)

7. K. Tokimatsu, and H.B. Seed, Journal of Geotechnical and Engineering, 113(8), 861-878, (1987)

8. H.B. Seed, I.M. Idriss, and I. Arango, Journal of Geotechnical Engineering, 109(3), 458-482, (1983)

9. P.M. Duku, J.P. Stewart, D.H. Whang, and R. Venugopal, Geotechnical Testing Journal, 30(5), 368-377, (2007)

10. P.M. Duku, J.P. Stewart, D.H. Whang, and E. Yee, Journal of Geotechnical and Geoenvironmental Engineering, 134 (8), 1073-1085, (2008)

11. E. Yee, J.P. Stewart, and F.P. Schoenberg, Soil Dynamics and Earthquake Engineering, 31(1), 25-32, (2011) 